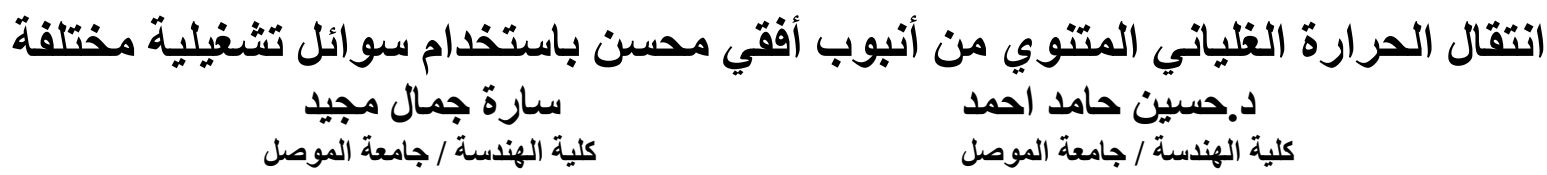

\title{
الخلاصة
}

في هذا البحث تم استقصاء عملي على الغليان الحوضي المتنوي من أنبوب أفقي منفرد حيث أستخدم الماء

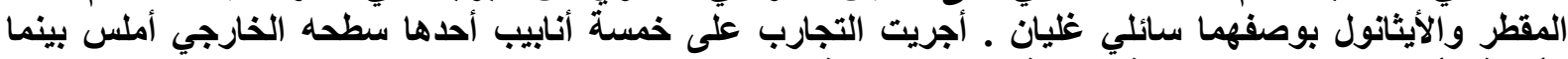

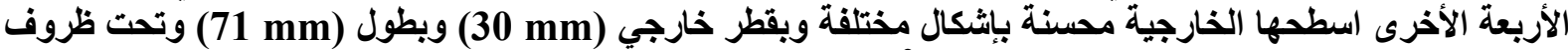

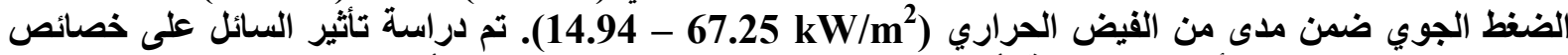

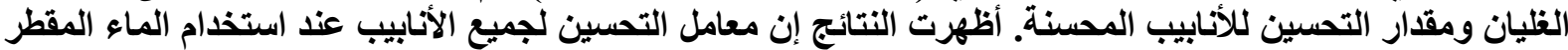

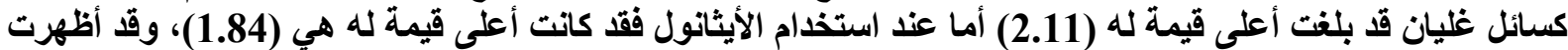

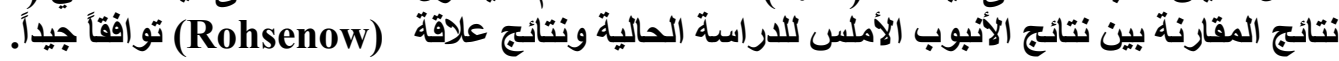
الكلمات الدالة : الغليان الحوضي المتنوي، التحسين، سائل الغليان.

\section{Nucleate Boiling Heat transfer From an Enhanced Horizontal Tube Using Different Working Liquids}

\author{
Dr. Hussain H .Ahmad \\ College of Engineering \\ University of Mosul
}

\author{
Sarah J. Majed
}

\begin{abstract}
In this research, an experimental investigation of nucleate pool boiling from a single horizontal tube has been done, with distilled water and ethanol as working liquids. The tests were carried out on five tubes; one of them has smooth external surface where the other four tubes have enhanced external surfaces of different finish with outer diameter $(30 \mathrm{~mm})$ and length $(71 \mathrm{~mm})$ under atmospheric pressure within the range of heat flux $\left(14.94-67.25 \mathrm{~kW} / \mathrm{m}^{2}\right)$. The effect of the liquid on the characteristics of boiling and amount of enhancement of enhanced tubes were studied. The results showed that the enhancement factor of all tubes has maximum value with distilled water as boiling liquid equal to (2.11). Where with ethanol, the maximum value was equal to (1.84) .The comparison between results of smooth tube in the present work and results of (Rohsenow correlation ) showed a good agreement.
\end{abstract}




\section{قائمة الرموز}

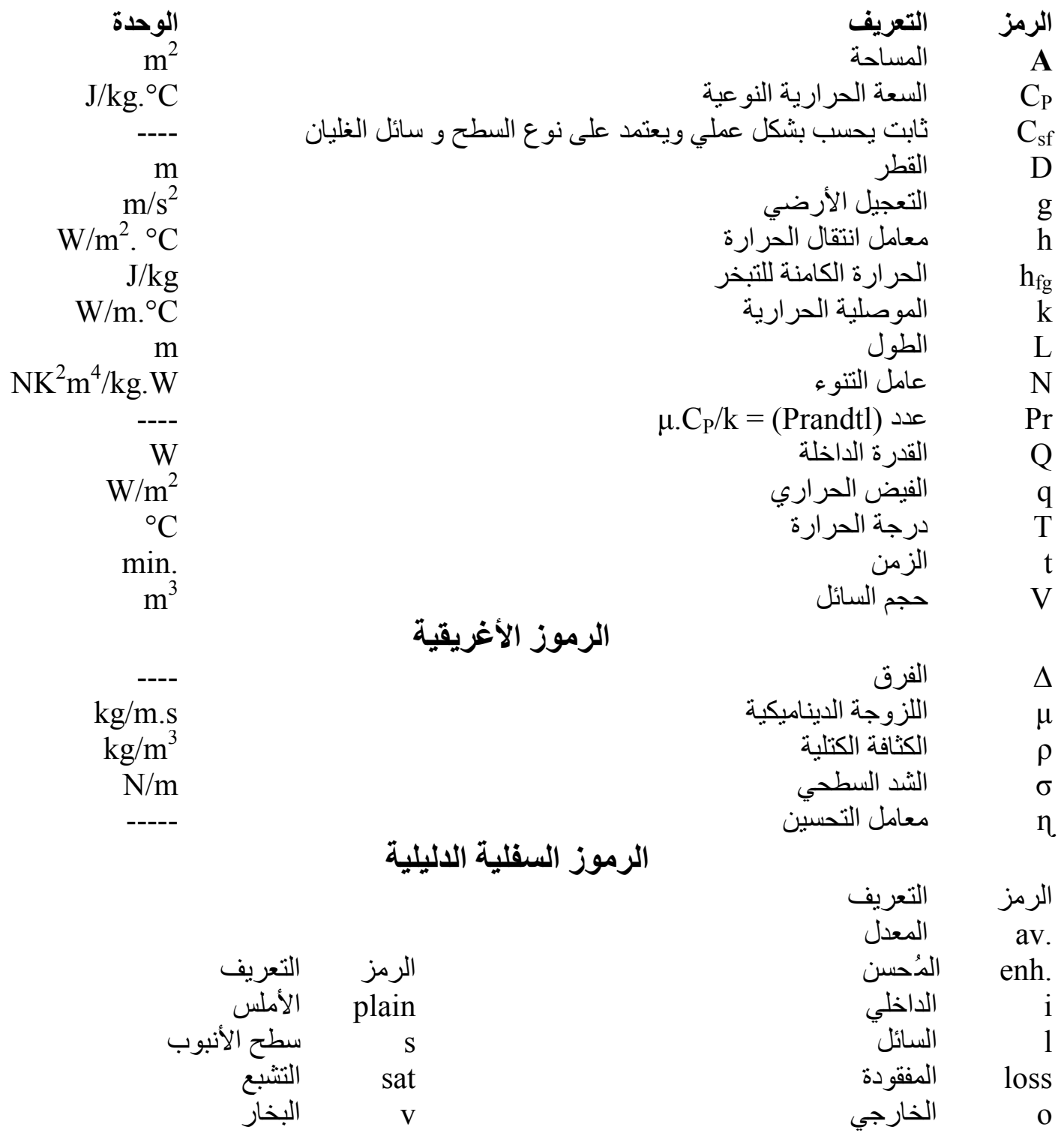

\section{1 (المقدمة}

يُعدّ انتقال الحر ارة الغلياني من الطرق ذات الكفاءة العالية لانتقال الحرارة ويحدث خلاله تغير في الطور من السائل

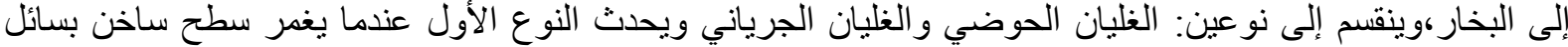

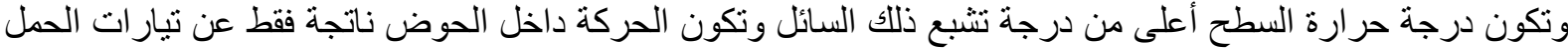

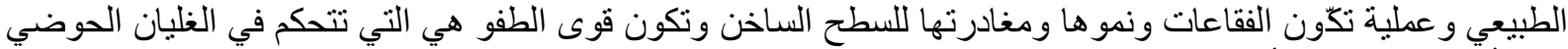

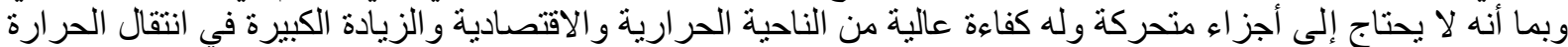

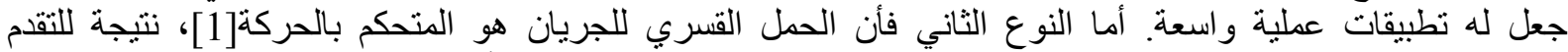

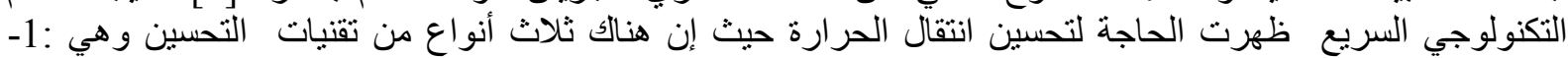

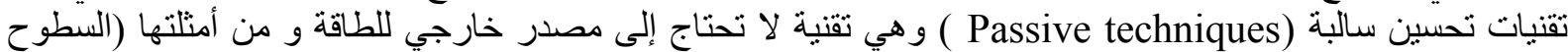
المعالجة والسطوح الممتدة والسطوح الخشنة والأنابيب الملفوفة وأدوات الثند السطحي وأدوات تدوير الجريان ).2 ـتقنيات 


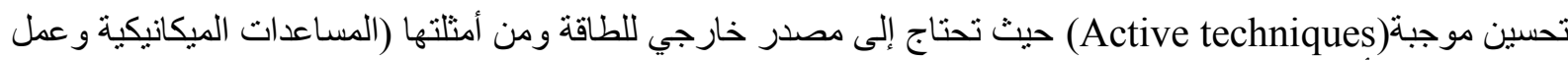
اهتز از للسطح أو للسائل وتسليط مجال الكتروني وطر ائق الحقن والامتصاص و إضافات للسائل).3-تقنيات تحسين مركبة ولتية (Compound techniques)

إن للأسطح المحسنة نطبيقات عملية واسعة في مجال المبادلات الحر ارية و الطير ان و الفضاء و التبريد الزبات الزمهريري

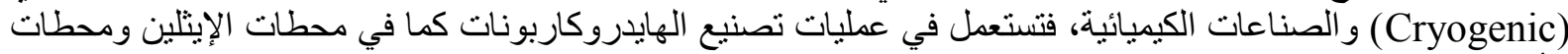

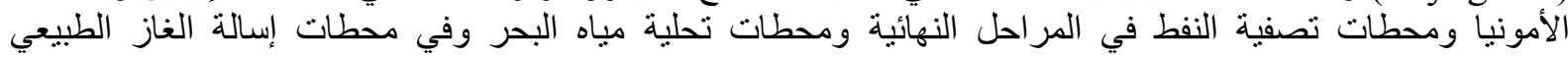

.[3](LNG)

أجرى الباحثان (Shou) و(Pao) [4] دراسة عملية لخصائص الغليان الحوضي لثناثة سوائل(الماء المقطر

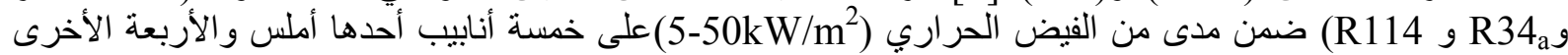

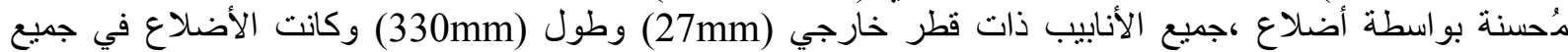

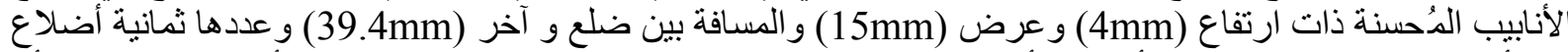

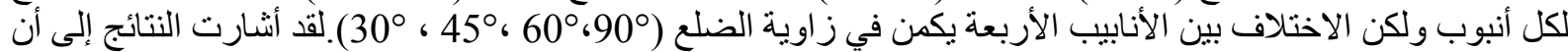

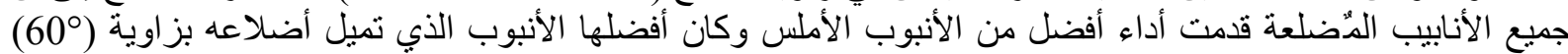

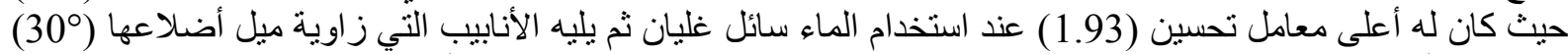

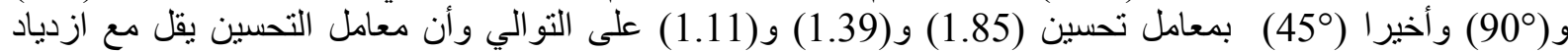

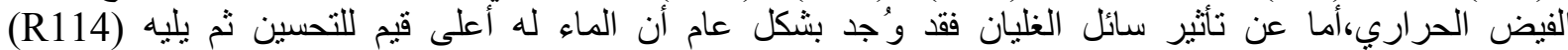
وأخيرأ)

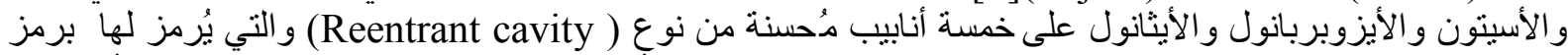

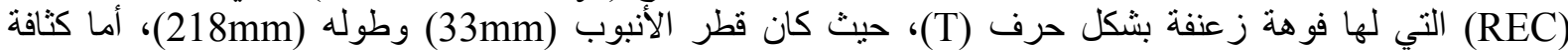
الزعانف فيه فكانت (1024fpm) ومُجوف من الاخل فئل لوضع مسخن كهربائي من نوع (33) (Cartridge heater) داخله

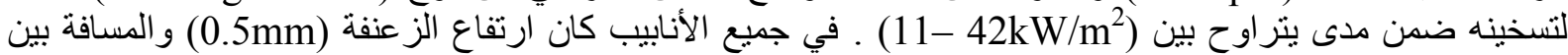

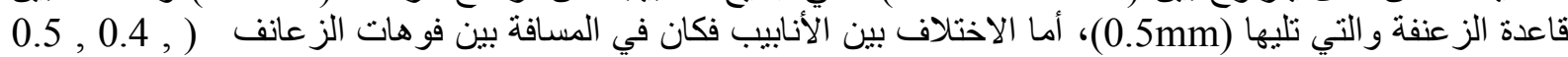

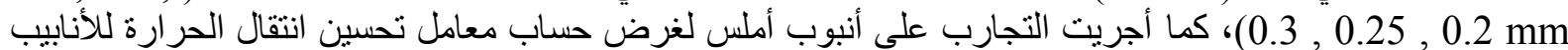

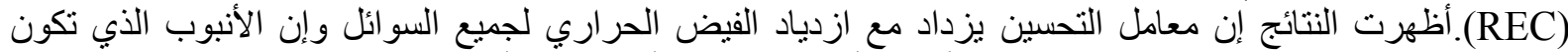

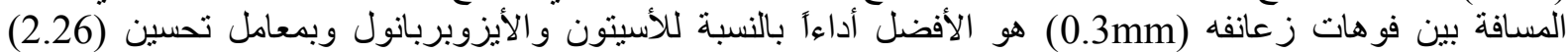

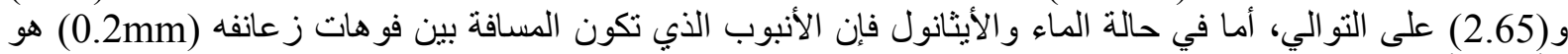

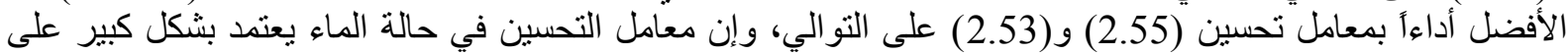

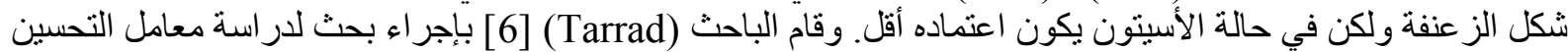

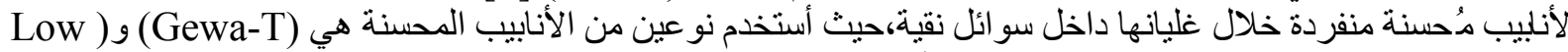

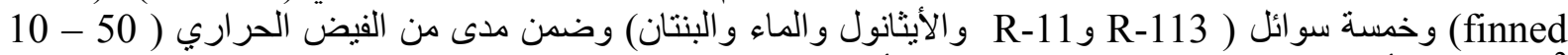

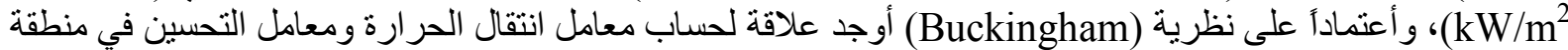

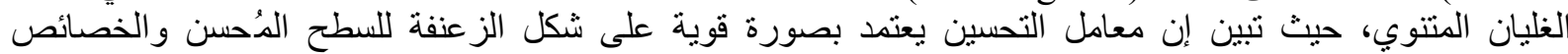
الفيزيائية لسائل الغليان وقد استنتج العلاقة الآتية:

$\eta=C_{S, F}\left(\frac{\rho_{1} h_{f g}{ }^{3 / 2}}{q}\right)^{m}\left(\frac{C_{p l} \sigma}{k_{1} h_{f g}{ }^{0.5}}\right)^{n}$

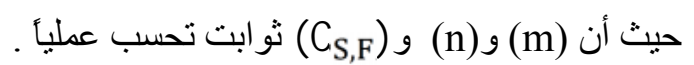

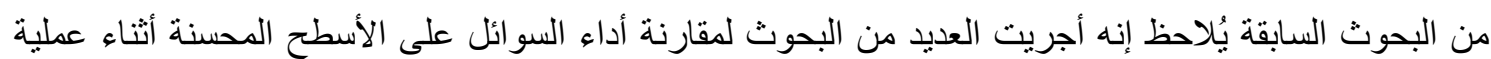

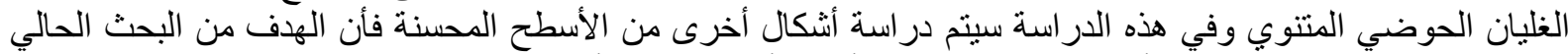

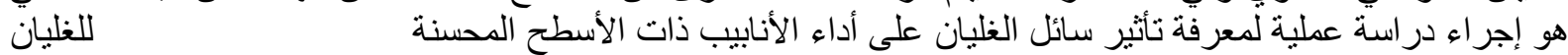

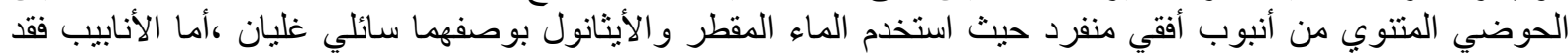

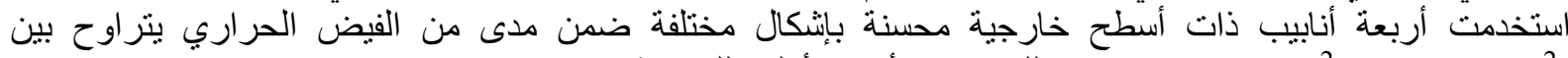
(14.94 kW/m² -67.25kW/m² ) 


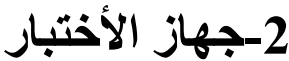

يتكون الجهاز من حوض اسطواني مصنوع من الفولاذ المقاوم للصدأ ذي قطر (140mm) وبطول (125mm)

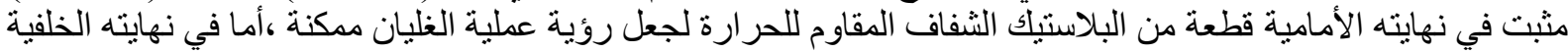

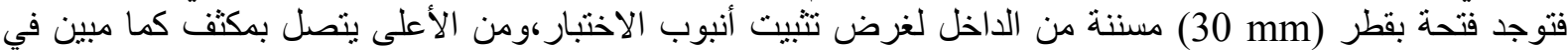

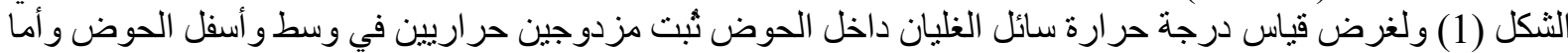

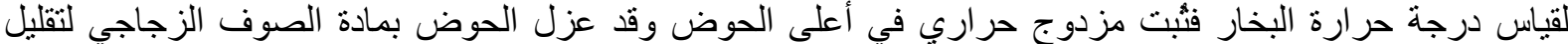

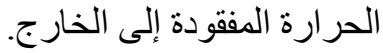

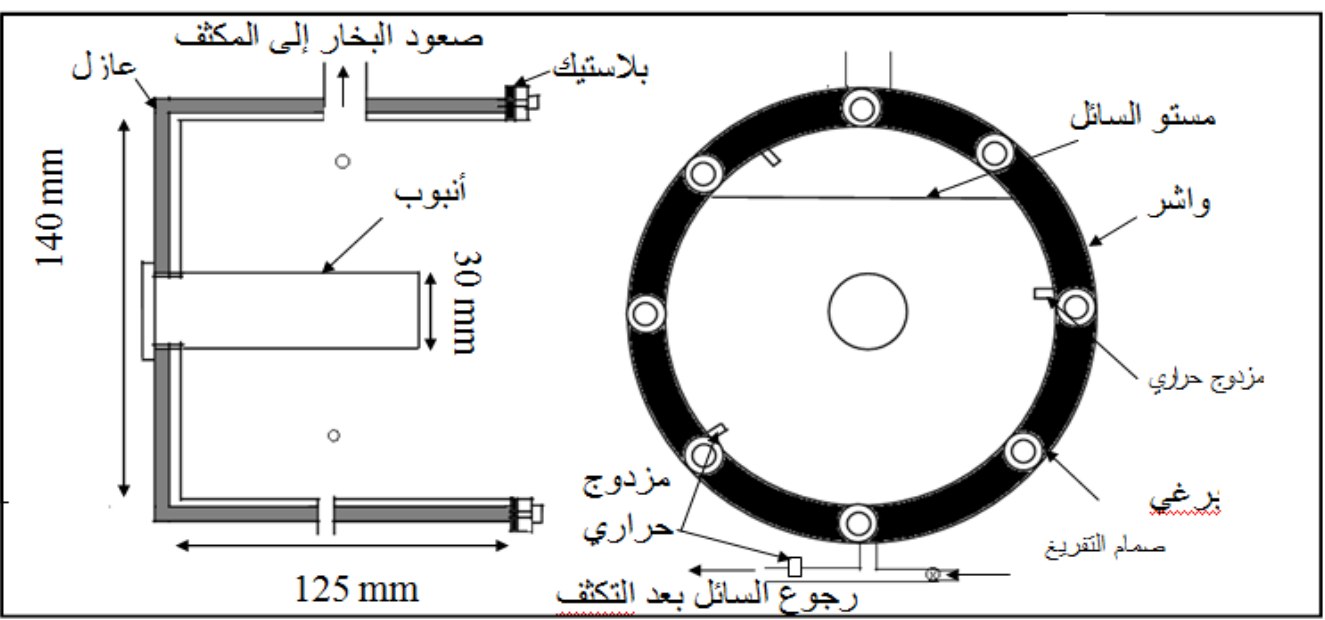

$$
\text { الثكل (1) : رسم توضيحي لحوض الغليان وبداخله أنبوب الاختبار ومو اقع المزدوجات الحرارية فيه }
$$

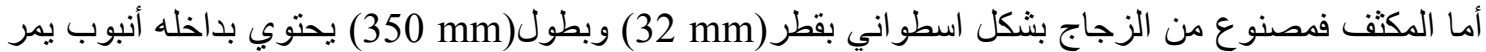

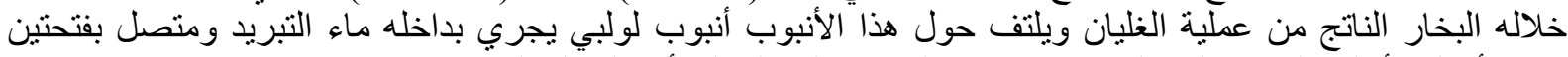

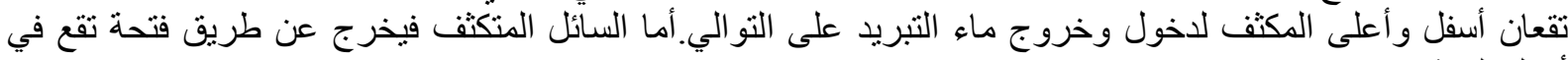
أسفل المكثف.

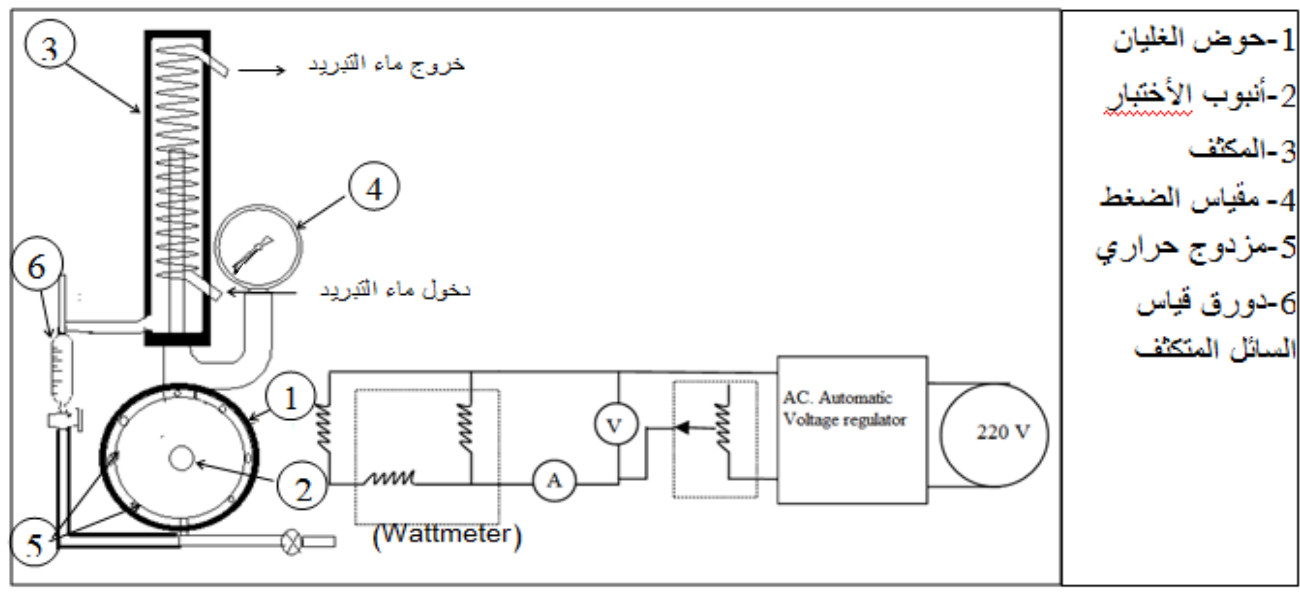

الثكل (2):رسم توضيحي للجهاز و الدائرة الكهربائية 


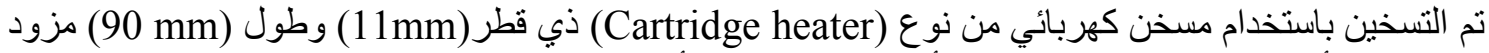

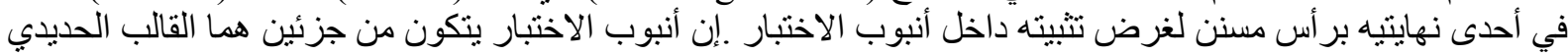

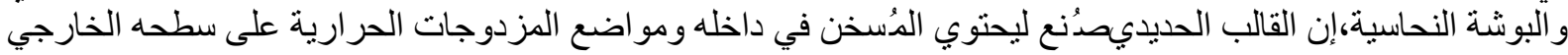

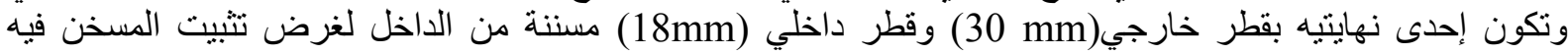

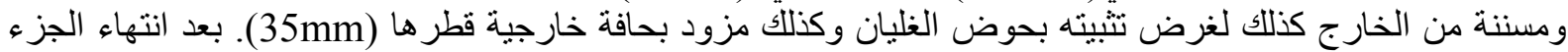

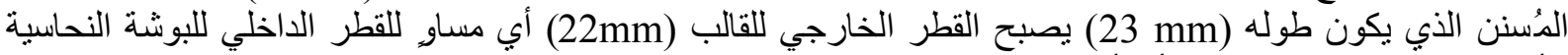

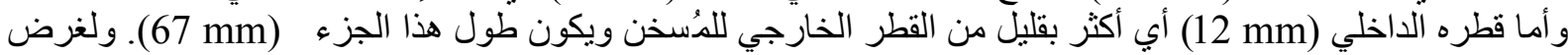

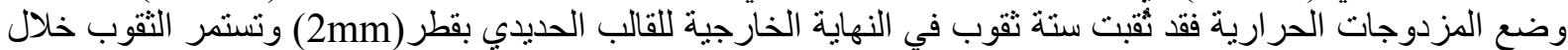

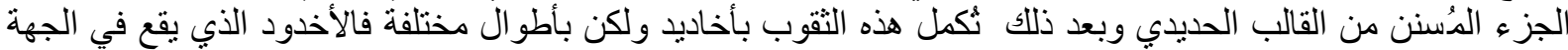

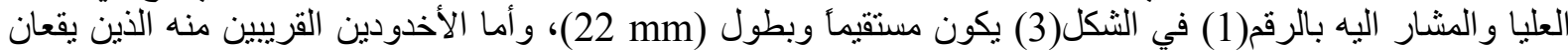

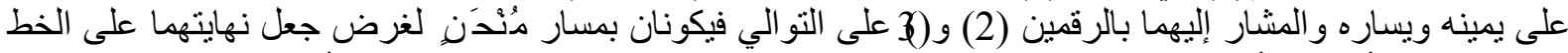

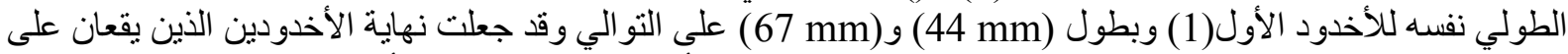

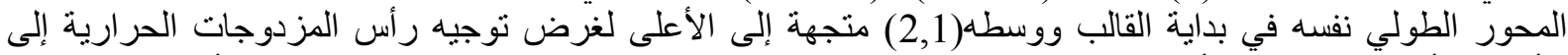

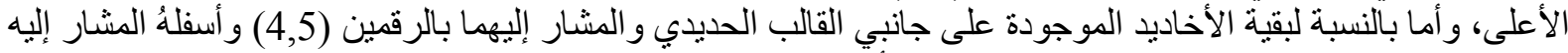
بالرقم (6) فيكونون بمسار مستقيم وبطول(67 mm) أي إلى نهاية القالب الحديدي.
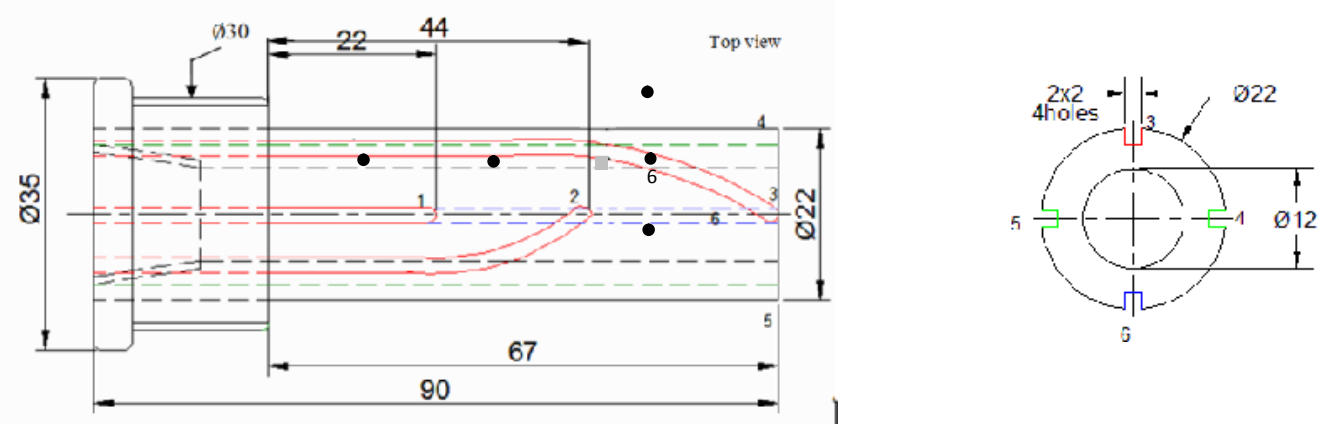

$$
\text { الثنكل (3): رسم توضيحي لمو اقع المزدوجات الحر ارية على سطح القالب الحديدي. }
$$

أما الجزء الثاني من الأنبوب فهو البوشة النحاسية التي ثُعدّ السطح الخارجي للأنبوب وتكون بقطر داخلي وخارجي

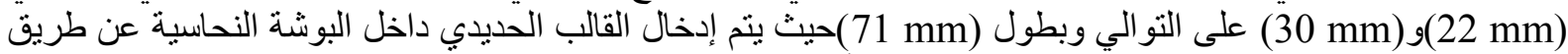

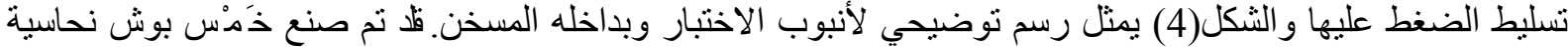

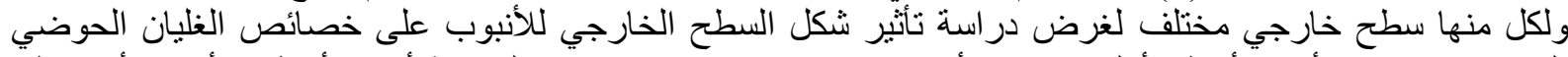

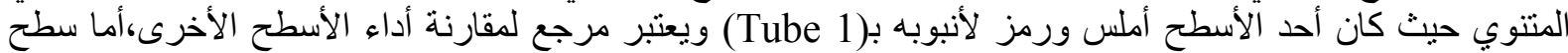

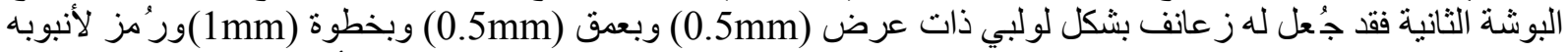

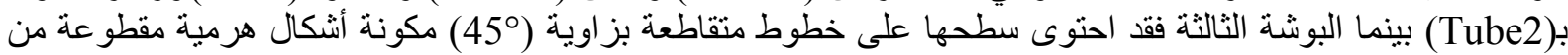

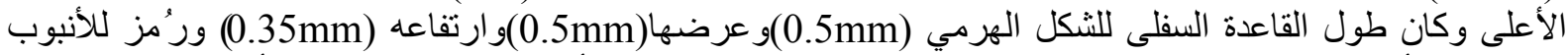

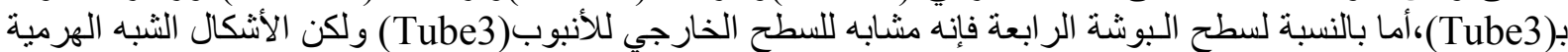

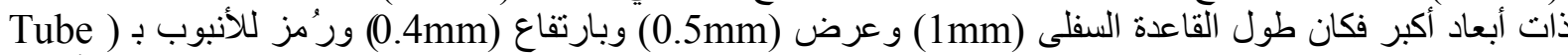

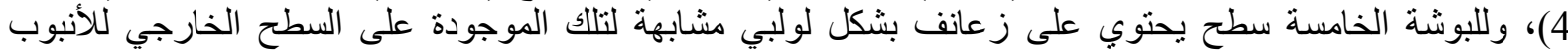

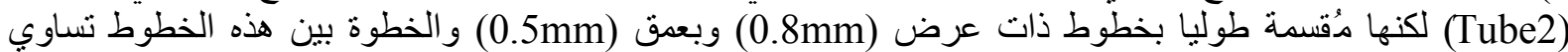

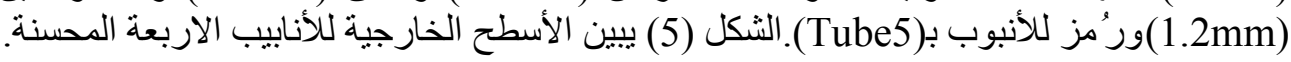




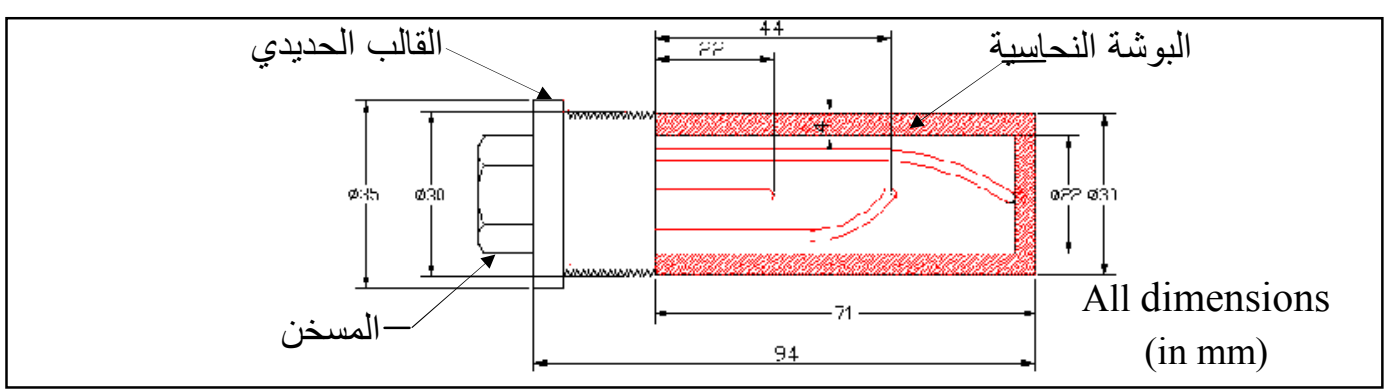

الثكل (4): رسم توضيحي لأنبوب الاختبار وبداخله المسخن.

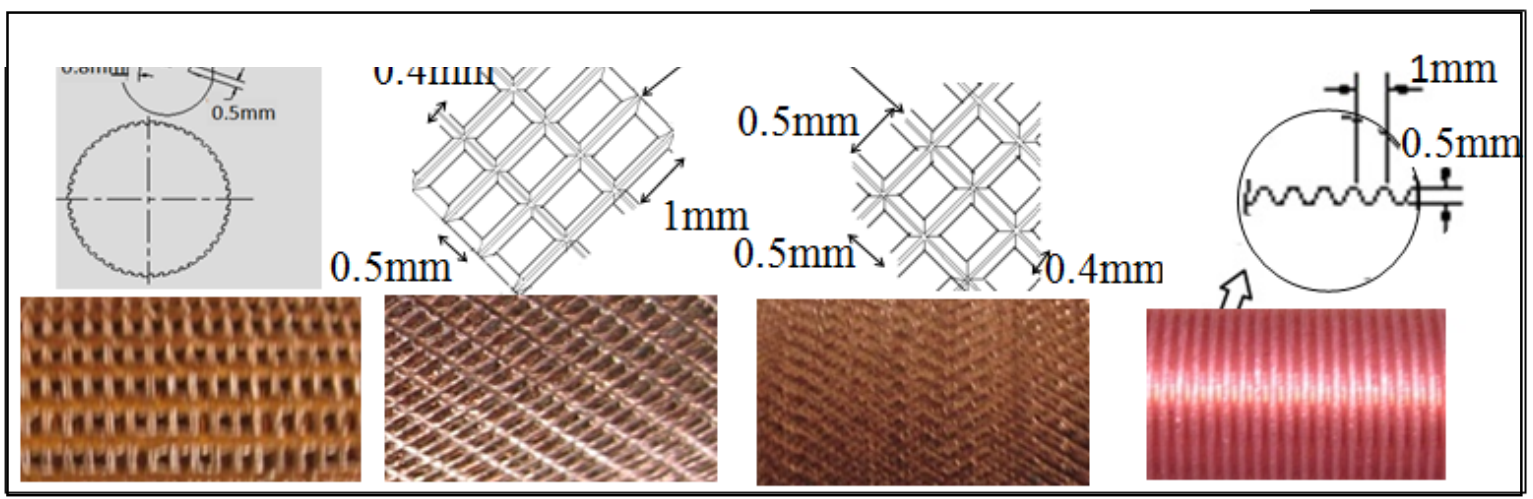

$$
\text { الثكل (5): الأسطح الخارجية للأنابيب الأربعة المحسنة. }
$$

أن الطاقة الكهربائية زودت باستخدام مثبت فولتية لتفادي التذبذب الحاصل في تجهيز هاومن ثم ثُغير القدرة الداخلة باستخدام مغير فولتية وينم قر اءتها عن طريق جهاز قياس القدرة (Wattmeter).

3-1ائتائج والمناقشة 3-1 تأثير شكل السطح الخارجي للأنبوب على الفرق بين درجة حرارة سطح الأنبوب ودرجة تثبع السائل

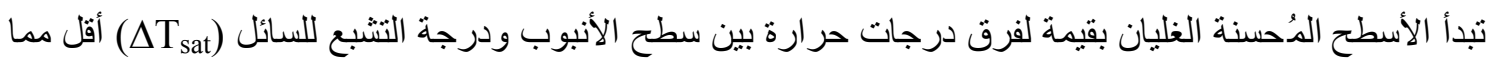

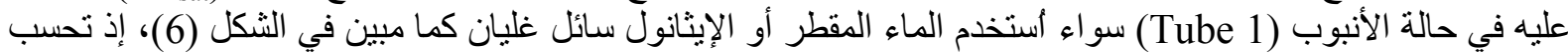

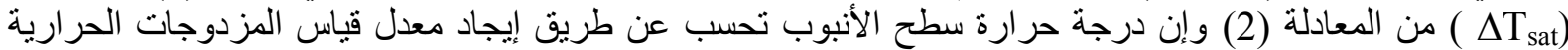

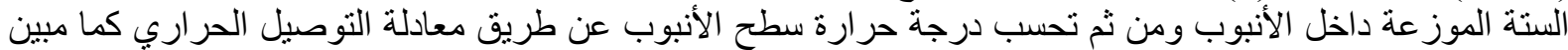

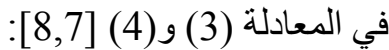

$\Delta \mathrm{T}_{\text {sat }}=\mathrm{T}_{\mathrm{s}}-\mathrm{T}_{\text {sat }}$

$\mathrm{T}_{\mathrm{av} .}=\frac{\sum \mathrm{T}}{6}$

$\mathrm{T}_{\mathrm{s}}=\mathrm{T}_{\mathrm{av}}-\mathrm{Q}\left(\ln \left(\mathrm{D}_{\mathrm{o}} / \mathrm{D}_{\mathrm{i}}\right) / 2 \pi \mathrm{Lk}\right)$

[10,9] أما الفيض الحر اري فيحسب من المعادلة (5)

$\mathrm{q}$
$=\frac{\mathrm{Q}}{\pi \mathrm{D}_{0} \mathrm{~L}}$ 

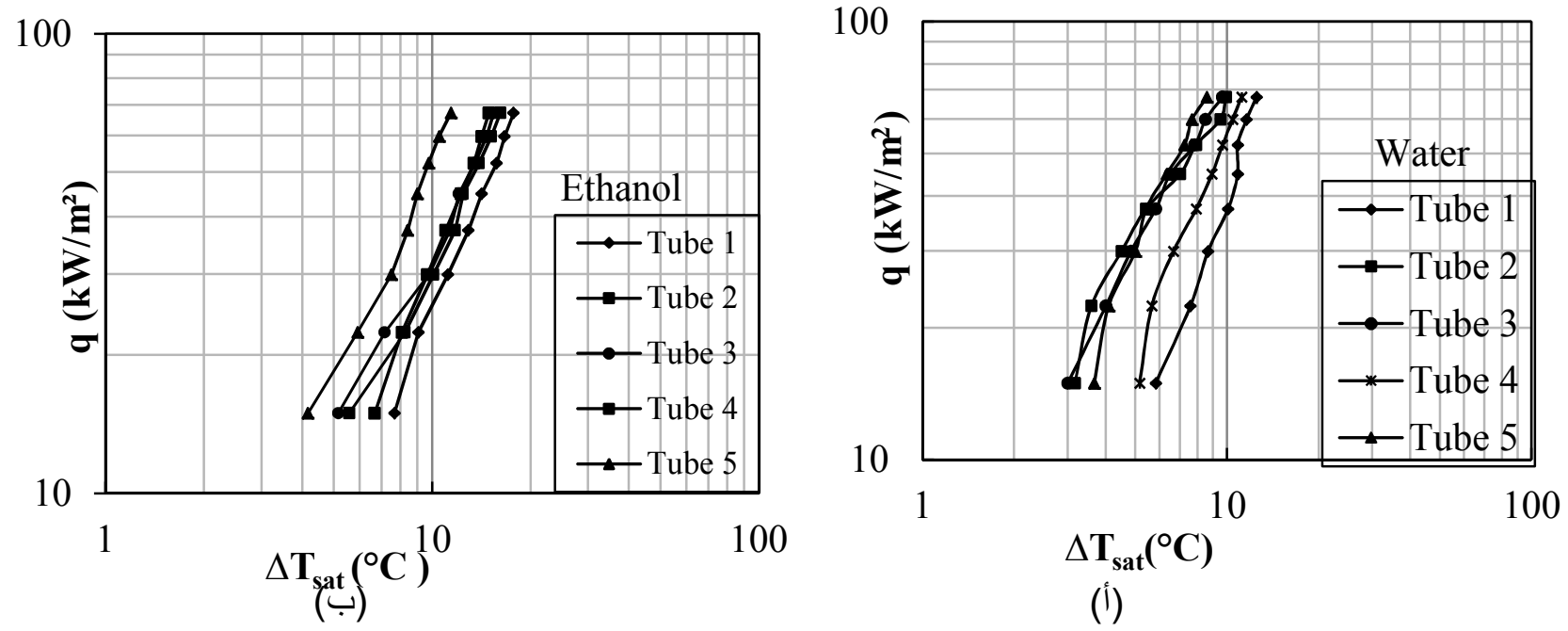

الثكل (6):تغير الفرق بين درجة حرارة سطح الأنبوب ودرجة تشبع السائل مع الفيض الحراري مقارنة بين جميع

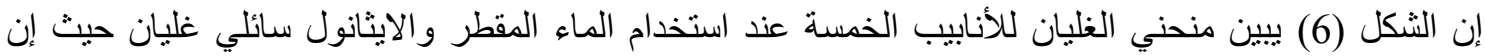

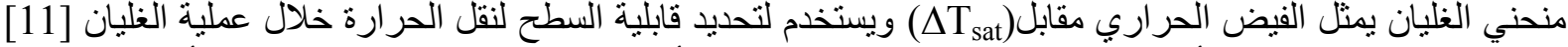

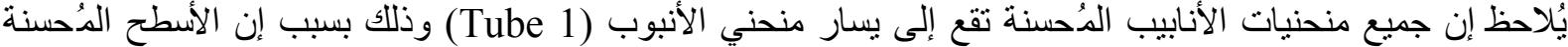

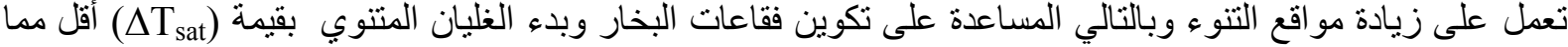

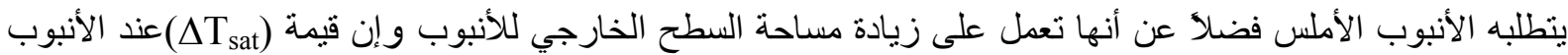

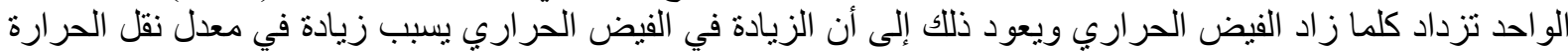

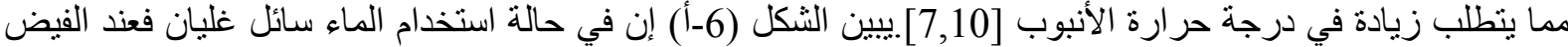

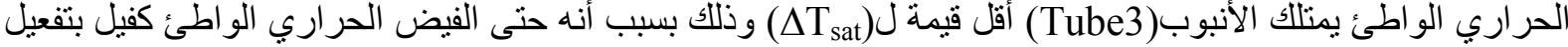

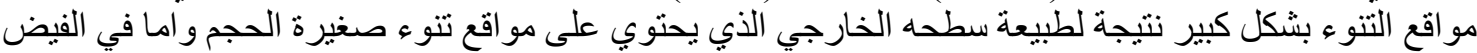

الحراري المتوسط فأن الأنبوب(Tube2) يمتلك أقل قيمة(

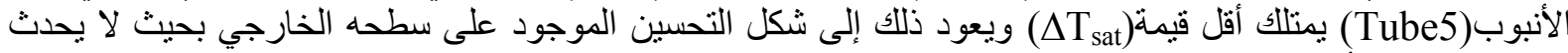

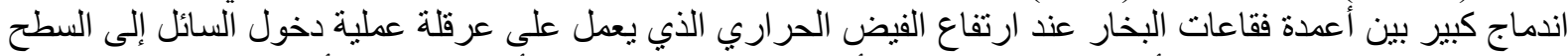

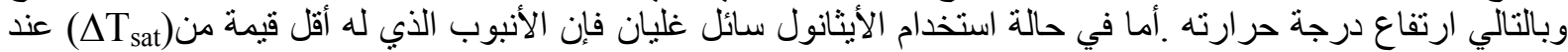

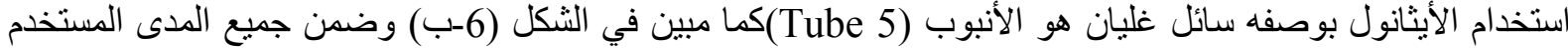
من الفيض الحراري ويعود ذللك إلى شكل التحسين الموجود على سطحه الخارجي ثم تنأتي الأنابيب (Tube3) و(Tube2)(أما أقلها تحسن هو الأنبوب (Tube 4).

\section{3-2 تأثير سائل الظليان على زمن الوصول إلى حالة الأستقرار}

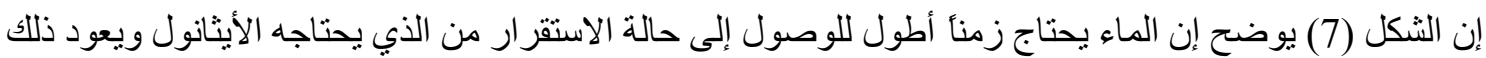

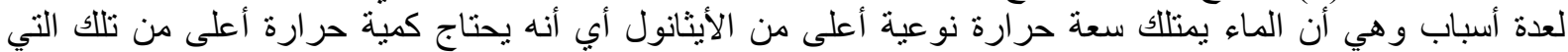

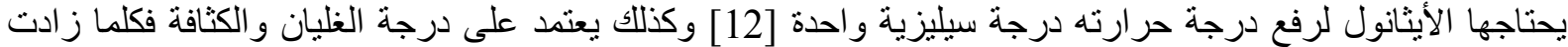

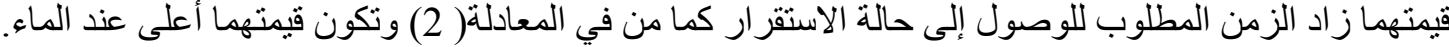

$\mathrm{t}=\rho * \mathrm{~V} * \mathrm{C}_{\mathrm{Pl}} * \frac{\left(\mathrm{T}_{\mathrm{sat}}-\mathrm{T}_{\mathrm{i}}\right)}{\mathrm{Q}-\mathrm{Q}_{\text {loss }}}$ 

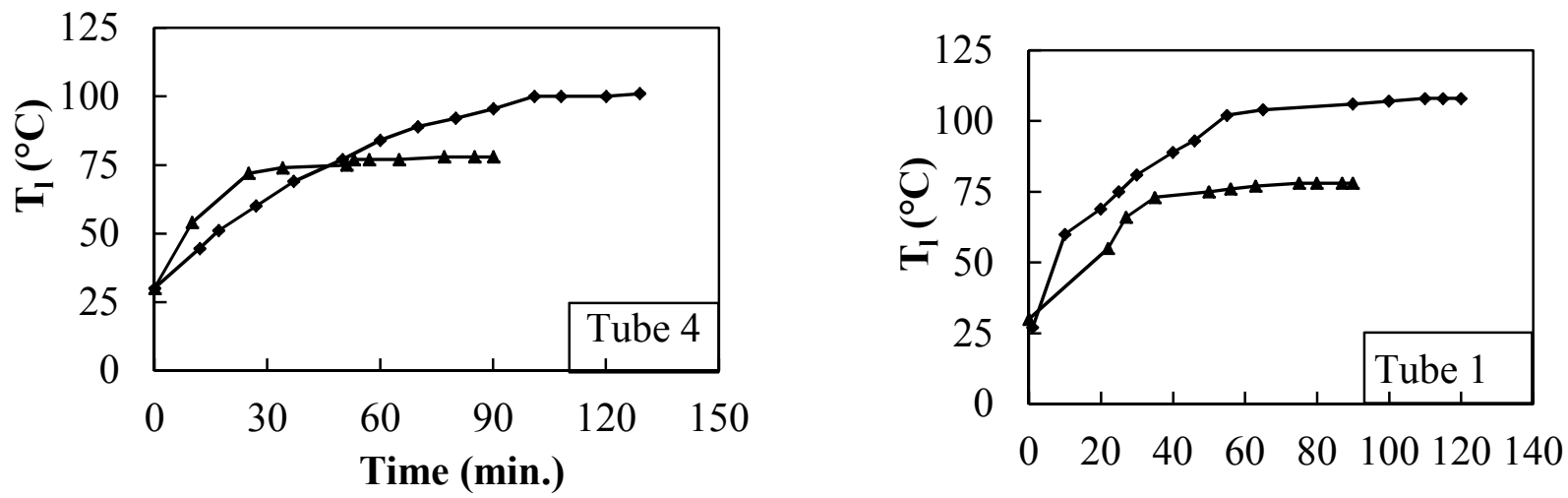

Time (min.)

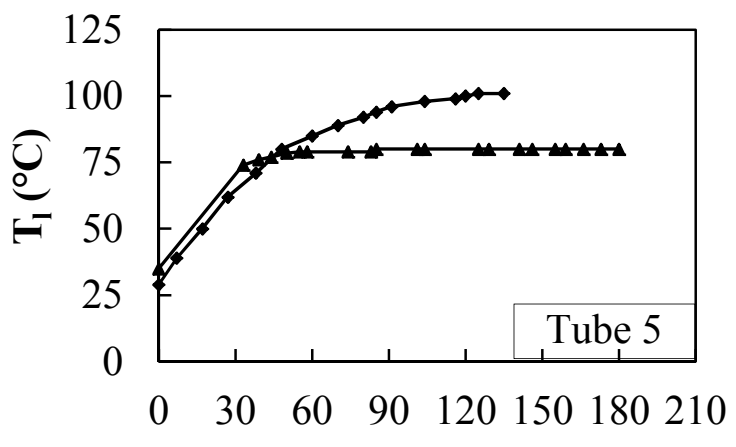

Time (min.)
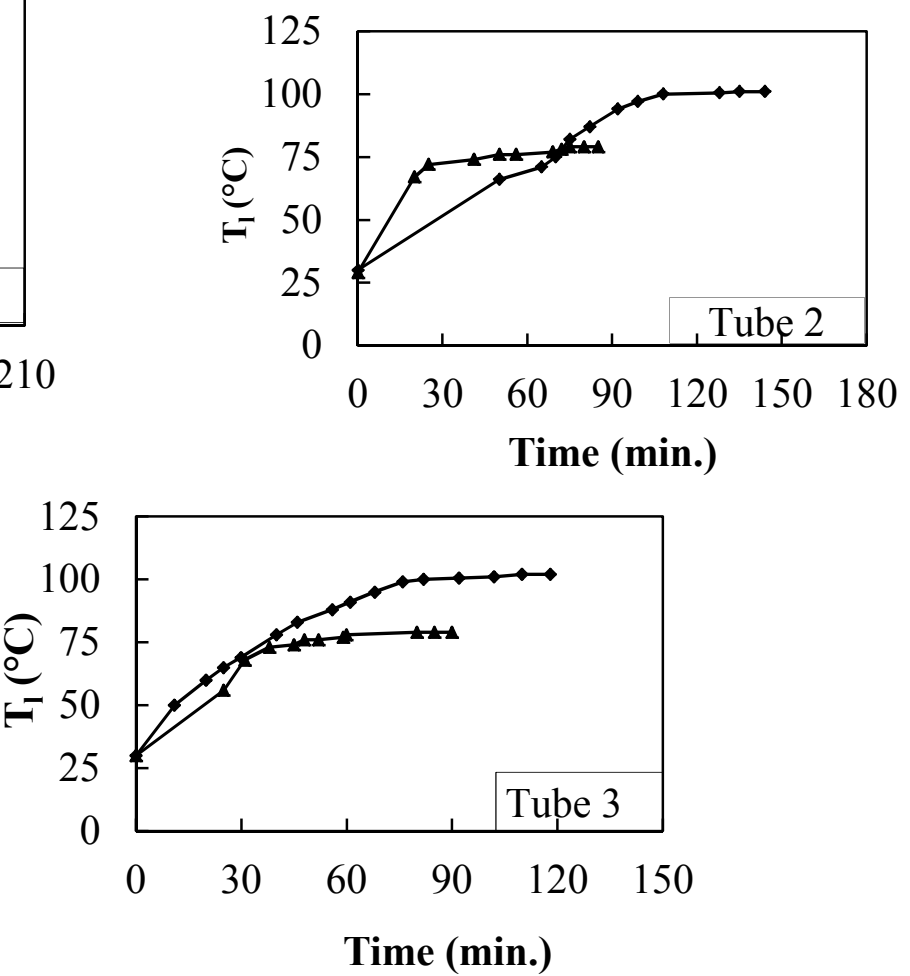

الثكل (7):مقارنة بين الفرق بين درجة حر ارة سطح الأنبوب ودرجة تشبع السائل مع زمن وصول الماء و الأيثانول إلى حالة الاستقر ار عند جميع الأنابيب .

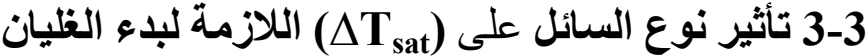

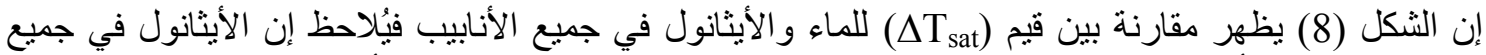

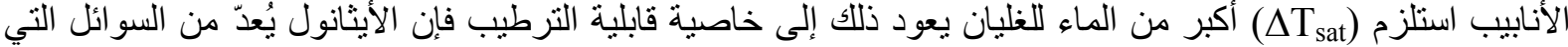

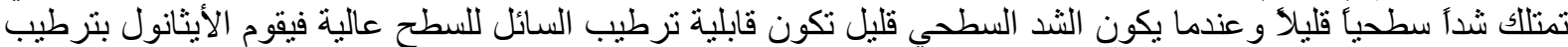
سطح الأنبوب بشكل جيد ولهذا تُغمر الفجوات الموجودة على سطح الأنبوب بالأيثانول مما يحتاج

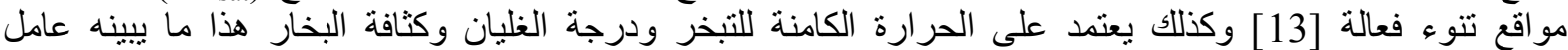

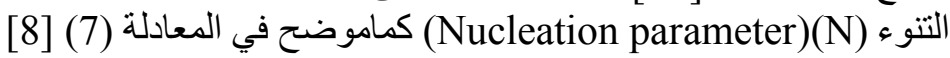

N $=\frac{\sigma \mathrm{T}_{\mathrm{sat}}}{\rho_{\mathrm{v}} \mathrm{h}_{\mathrm{fg}}}$ 
أحمد: انتقال الحرارة الغلياني المتنوي من أنبوب أفقي محسن باستخدام سوائل تشغيلية مختلفة
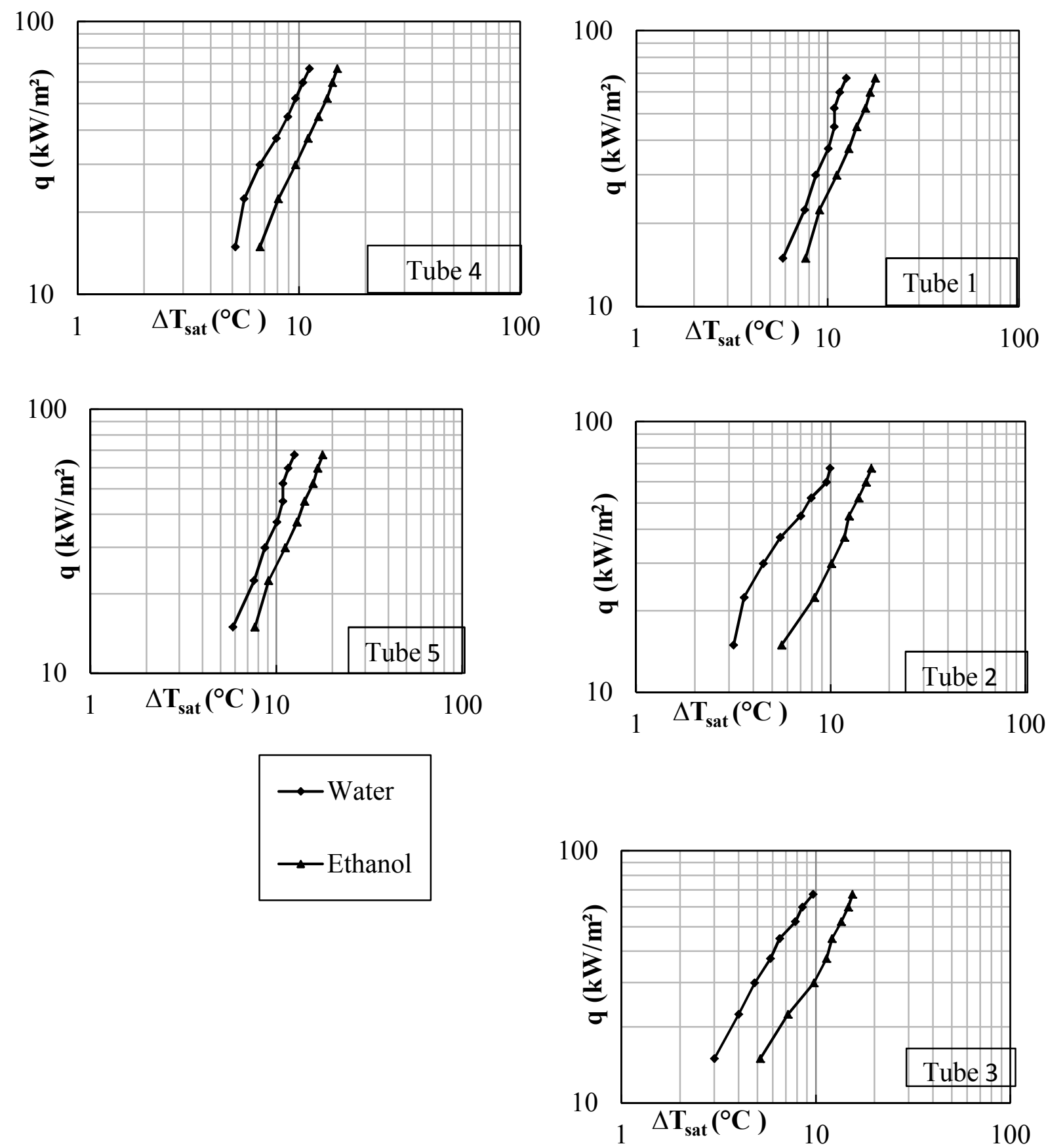

الشكل (8): تغير الفرق بين درجة حر ارة سطح الأنبوب ودرجة سائل الغليان مع الفيض الحر اري مقارنة بين الماء و الأيثانول: تلغيرل

\section{3-4 تأثير السائل المُستخدم على معامل انتقال الحرارة}

إن دراسة الغليان المتنوي تتطلب حساب معامل انتقال الحرارة لأنه العامل المهح في تصميم أجهزة انتقال الحرارة إنهاة

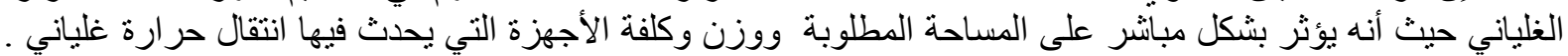

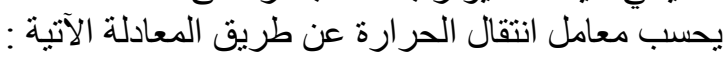

$\mathrm{h}=\frac{\mathrm{q}}{\Delta \mathrm{T}_{\mathrm{sat}}}$ 


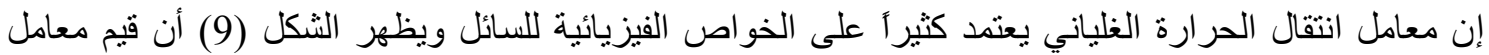

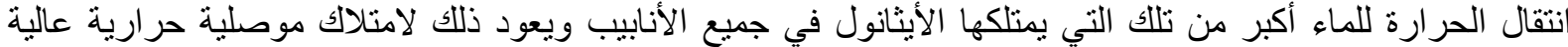

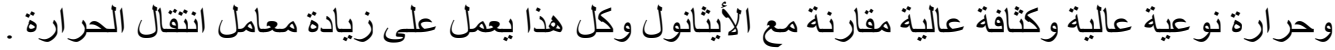
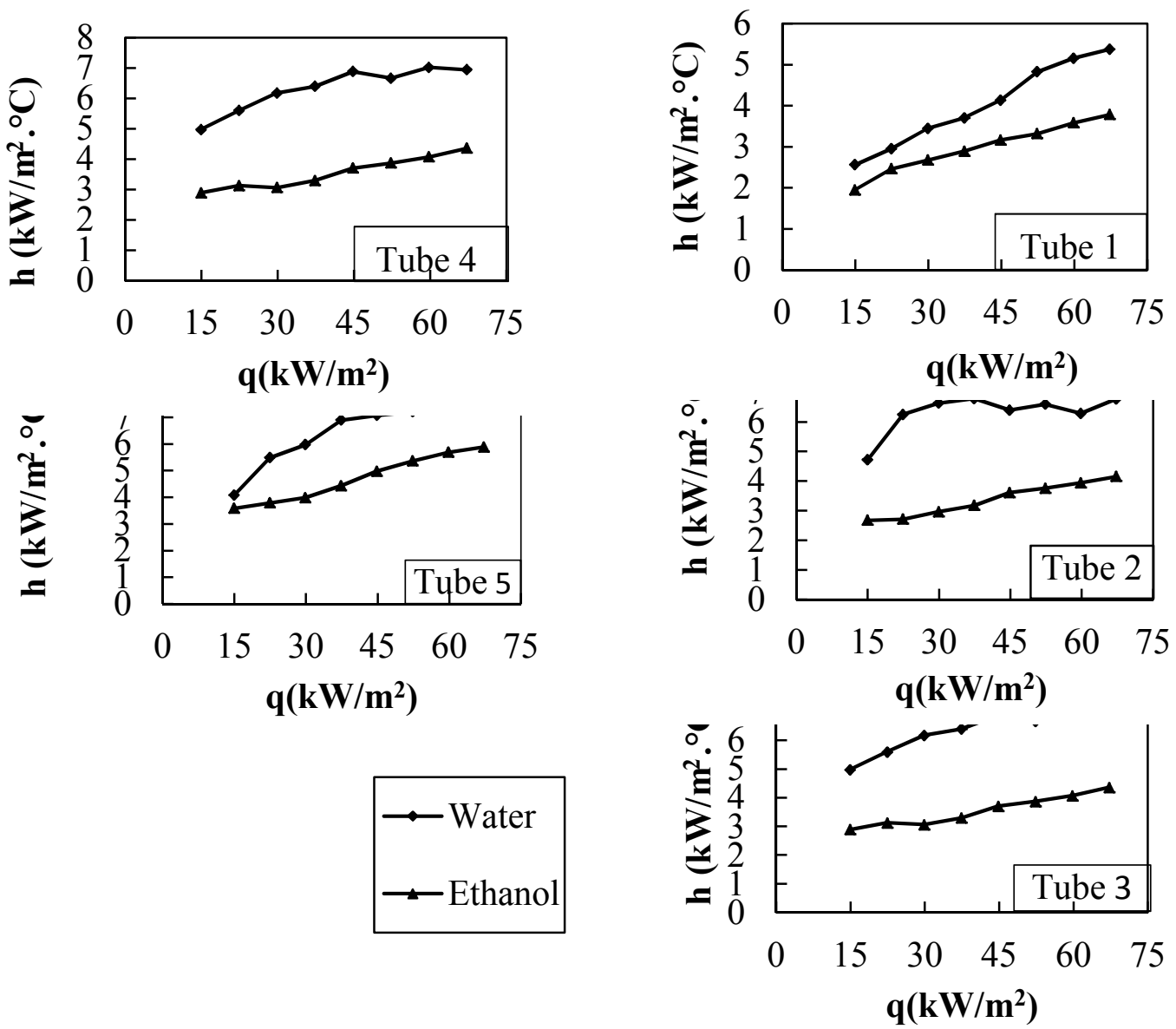

الثكل(9):مقارنة بين معامل انتقال الحرارة للماء وللأيثانول عند جميع الأنابيب.

3-5 تأثير نوع سائل الظليان على معامل التحسين

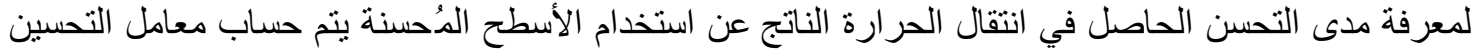

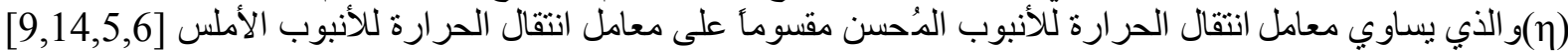
كما مبين في المعادلتين الآتيتين :

$\eta=\frac{\mathrm{h}_{\text {enh. }}}{\mathrm{h}_{\text {plain }}}$

$\eta=\frac{\Delta T_{\text {Plain }}}{\Delta T_{\text {enh }}}$

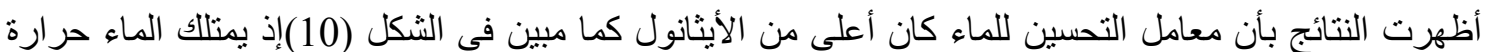

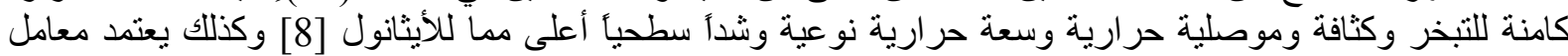
التحسين على معامل التقاء السطح بالسائل (C) 
أحمد: انتقال الحرارة الغلياني المتنوي من أنبوب أفقي محسن باستخدام سوائل تشغيلية مختلفة
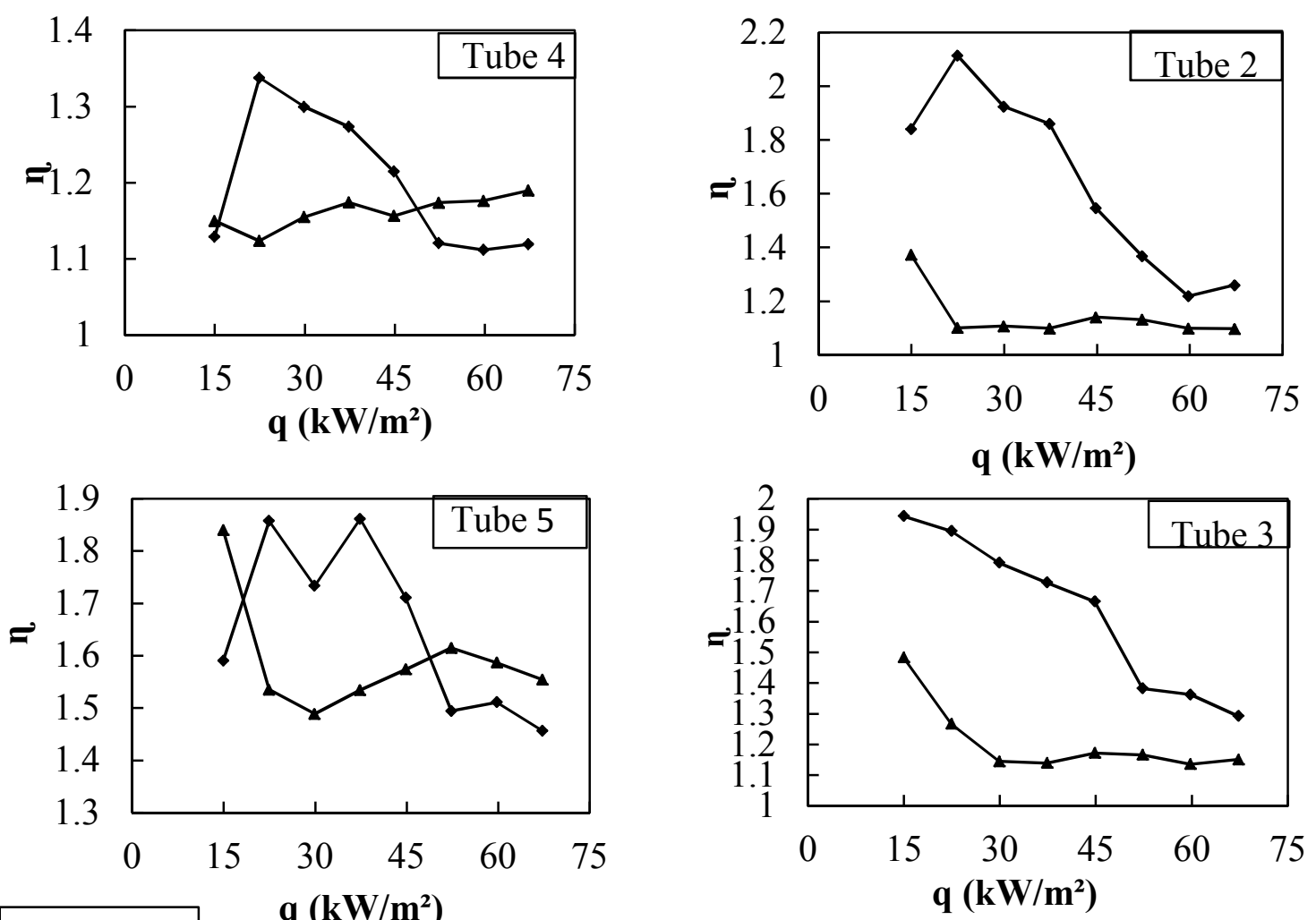

$\rightarrow$ Water
$\rightarrow$ Ethanol

الثكل (10): تأثير سائل الغليان على معامل التحسين للأنابيب المحسنة

3-6 مقارنة نتائج البحث الحالي مع علاقة (Rohsenow)

إن مقارنة النتائج المستحصلة من البحث الحالي للأنبوب الأملس (Tube 1) مع علاقة (Rohsenow)

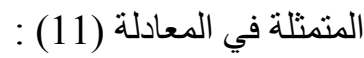

$\Delta \mathrm{T}_{\mathrm{sat}}=\mathrm{C}_{\mathrm{sf}} \frac{\mathrm{h}_{\mathrm{fg}}}{\mathrm{CPl}_{\mathrm{Pl}}}\left|\frac{\mathrm{q}}{\mu_{\mathrm{l}} \mathrm{h}_{\mathrm{fg}}}\left(\frac{\sigma}{\mathrm{g}\left(\rho_{1}-\rho_{\mathrm{v}}\right)}\right)^{1 / 2}\right|^{\mathrm{n}} \operatorname{Pr}_{\mathrm{l}}^{\mathrm{m}+1}$

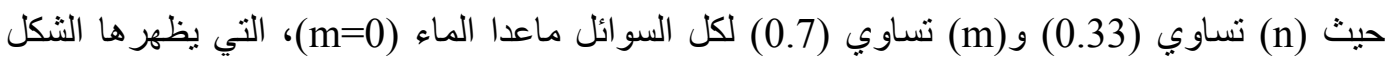

(11) ييين أن هناك تو افق كبير بين نتائج البحث الحالي سواءٌ عند استخدام الماء المقطر او الايثانول سائل غليان و النتائج

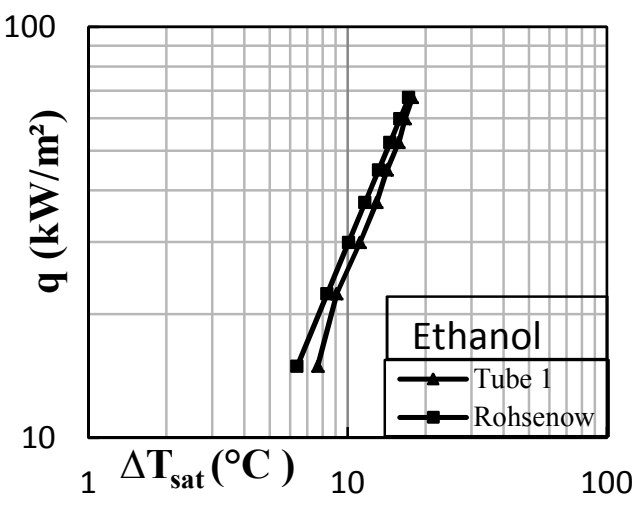

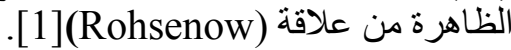

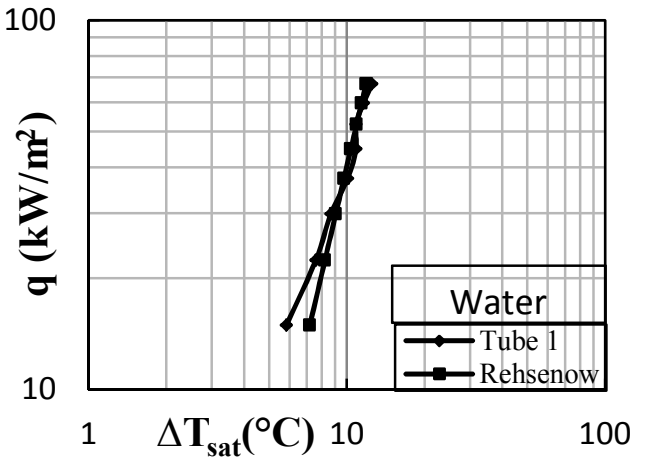

الثنكل (11): مقارنة نتائج البحث الحالي مع عالهه (Rohsenow). 
1- Tong, L. S. and Tang, Y. S., "Boiling heat transfer and two phase flow ", Second Edition, Published by Taylor and francis (1997) .

2- Bergles ,A.E. "High flux processes through enhanced heat transfer" the 5th International Conference on Boiling Heat Transfer, Montego Bay, Jamaica, (2003).

3- Shekriladze, I.G.," Boiling Heat Transfer: Mechanisms, Models, Correlations and the Lines of Further Research", The Open Mechanical Engineering Journal, Vol. 2, (2008), pp 104-127. 4- Shou, S.H., Pao, T.H. "Nucleate boiling characteristics of R-1 14, distended water $\left(\mathrm{H}_{2} 0\right)$ and R-134a on plain and rib-roughened tube geometries", International Journal heat mass transfer, Vol. 37, No3, (1994), pp 1423- 1432

5- Rajulu, K.G., Kumar, R., Mohanty, B., Varma, H. K., " Enhancement of nucleate pool boiling heat transfer coefficient by reentrant cavity surfaces ", International Journal of Heat Mass Transfer, Vol. 41, (2004),pp. 127-132.

6- Tarrad, A.H. , "A simplified correlation for the prediction of nucleate pool boiling performance of single integral enhanced tubes boiling pure liquids at atmospheric pressure", Journal of Engineering and Development, Vol. 11, No. 3, (2007),pp.131-148 .

7- Bhaumik, S. , Ayarwal, V.K. , Gupta, S. " A generalized correlation of nucleate pool boiling of liquids ", Indian journal of chemical technology, Vol. 11,(2004), pp 719-725.

8- Perry, G.D." Effect of oil on the onset of nucleate pool boiling of R-124 from a single horizontal tube ",M.SC Thesis, Naval postgraduate school Monterey, California ,(1993) .

9- Tarrad, A.H. and Burnside, B. M., " Pool boiling tests on plain and enhanced tubes using a wide-bolling-range mixture" Journal of Experimental heat transfer, vol. 6, (1993) , pp. 8396.

10- Bergles, A. E. , Jensen, M. K. , Somerscales, E. F., Curcio, L. A., Trewin, R.R., "Enhanced shell-and-tube heat exchangers for the power and process industries" ,Rensselaer Polytechnica Institute Troy, New York (1994).

11- Sarafraz, M.M., Peyghambarzadeh, S.M., Fazel, A., " Experimental studies on nucleate pool boiling heat transfer to ethanol/MEG/DEG ternary mixture as a new coolant" Islamic Azad University, Mahshahr, Iran (2012) 
12- Witharana, S., "Boiling of refrigerants on enhanced surfaces and boiling of nanofluids", M.SC. Thesis, The Royal Institute of Technology, (2003) .

13- Shou, S.H. , Pao, T.H. "Nucleate boiling characteristics of R-1 14, distended water $\left(\mathrm{H}_{2} 0\right)$ and R-134a on plain and rib-roughened tube geometries", International Journal heat mass transfer, Vol. 37, No3, (1994), pp 1423- 1432

14- Jung, D., An, K., Park, J., " Nucleate boiling heat transfer coefficients of HCFC22,HFC134a, HFC125, and HFC32 on various enhanced tubes", International Journal of Refrigeration, Vol. 27, (2004), pp. 202-206. 